\title{
Intestinal microbiome-gut-brain axis and irritable bowel syndrome
}

\author{
Gabriele Moser (D) C Camille Fournier · Johannes Peter
}

Received: 2 March 2017 / Accepted: 26 July 2017 / Published online: 8 September 2017 (C) The Author(s) 2017. This article is an open access publication.

Summary Psychological comorbidity is highly present in irritable bowel syndrome (IBS). Recent research points to a role of intestinal microbiota in visceral hypersensitivity, anxiety, and depression. Increased disease reactivity to psychological stress has been described too. A few clinical studies have attempted to identify features of dysbiosis in IBS. While animal studies revealed strong associations between stress and gut microbiota, studies in humans are rare. This review covers the most important studies on intestinal microbial correlates of psychological and clinical features in IBS, including stress, anxiety, and depression.

Keywords Stress $\cdot$ Psyche $\cdot$ Depression · Anxiety $\cdot$ Enterotype

\section{Intestinale Mikrobiom-Darm-Hirn-Achse und Reizdarmsyndrom}

Zusammenfassung Psychische Komorbidität ist häufig bei Reizdarmsyndrom (RDS). Die aktuelle Forschung weist auf eine Rolle des intestinalen Mikrobioms bei der viszeralen Hypersensitivität, bei Angst und Depression hin. Eine Steigerung der Krankheitssymptomatik bei psychischem Stress wurde beschrieben. Wenige klinische Studien haben versucht, Muster einer Dysbiose für RDS zu identifizieren. Während Tierstudien eine starke Assoziation zwischen Stress und Darm-Mikrobiom aufdeckten, sind Studien beim Menschen rar. Diese Übersicht umfasst die wichtigs-

Univ. Prof. Dr. G. Moser, MD $(\bowtie) \cdot$ C. Fournier, MD

J. Peter, M.Sc

Division of Gastroenterology and Hepatology, Department

of Internal Medicine III, Medical University of Vienna,

Waehringer Guertel 18-20, 1090 Vienna, Austria

gabriele.moser@meduniwien.ac.at ten Studien über Zusammenhänge zwischen dem intestinalen Mikrobiom und psychischen und klinischen Aspekten bei RDS, inklusive Stress, Angst und Depression.

Schlüsselwörter Stress · Psyche - Depression · Angststörung · Enterotyp

The intestinal microbiome, stress and the gut-brain axis

The human intestinal system is home to about 100 trillion microbes, mainly of bacterial origin [1]. Research has ascertained a critical relevance of gut bacteria for health and disease [2, 3], and there is strong evidence suggesting they can affect emotion processing and stress coping. The concept of a gut-brain-microbiota axis connects the psyche and nervous system with the intestine, its inhabitants and its metabolic, neuroendocrine, and immune functions [4, 5].

Gut microbiota sets developmental conditions for hypothalamic-pituitary-adrenal axis (HPA-axis) maturation $[6,7]$. Together with an impact on brain circuits of social cognition, reward, and emotion processing, this indicates a relevance for resilience and behavioral adaption to stress $[8,9]$. Vice versa, stress can decrease the diversity and alter the composition of the gut microbiome [10, 11]. Early life stress induced by maternal separation leads to lifelong alterations in microbial composition, HPA axis functioning and visceral hypersensitivity in rodents. This has been proposed as an animal model of irritable bowel syndrome (IBS) [12-14]. Loss of intestinal epithelial barrier function is another focus of research on the relationship between stress and microbiota. Suspected to provoke inflammatory cascades, autoimmunity, and pain, "leaky gut" can be both cause and consequence of psychological stress [15-17]. 
Intestinal microbiome, inflammation and psychological disorders

Another strong link between intestinal microbiota and psychological health is based on the "inflammation hypothesis" of psychiatric disease [18, 19]. Bacterial pathogens can initiate inflammatory reactions [20] or modulate inflammatory processes via metabolites (e. g., short-chain fatty acids (SCFAs)) [21, 22]. Furthermore, gut bacteria are involved in the metabolism of key neurotransmitters such as serotonin [5], and seem to intervene in the turnover of neuronal growth factors related to cognition and brain health, e. g. brain-derived neurotrophic factor (BDNF) [23, 24]. Microbiota-dependent effects on mouse reward circuitry [25], and probiotics associated alterations of emotion-processing brain activity in humans [26] have been reported.

Behavioral alterations (anxiety and depression-like behavior) by manipulations of the microbiome were demonstrated in seminal animal studies [27-29]. Recently, socially avoidant behavior, paralleled by decreased myelinization in the prefrontal cortex, was ascribed to bacteria-mediated epigenetic modulations in mice [30]. In humans, Lin and colleagues [31] found significant differences in the composition of the gut microbiome between patients suffering from major depressive disorders and controls, displaying a dysbalanced Firmicutes to Bacteroidetes ratio and higher abundance of Streptococcus, Klebsiella, Prevotella, and Clostridium XI in depressive patients.

Those results contrast with the findings of Jiang and colleagues [32]. Although reporting the same higher Firmicutes to Bacteroidetes ratio, they observed different associations between taxa and major depressive disorder. Faecalibacterium was inversely correlated with symptoms severity, and an association between Clostridium cluster XIV and BDNF serum concentrations was observed.

In healthy women, however, no association between microbial features and psychological parameters could be found [33]. Further studies will have to determine the precise role of the microbiome within the range of normal behavior to pathophysiology in psychiatric illness.

\section{Irritable bowel syndrome and microbiome-brain interaction}

Irritable bowel syndrome (IBS) is a functional gastrointestinal disorder with an estimated prevalence in the general population of 5 to $20 \%$ [34]. IBS is defined with following diagnostic criteria (ROME IV) [35]: recurrent abdominal pain, on average at least one day per week in the last 3 months. The pain should be related to defecation and associated with a change in frequency and in form (appearance) of stool.

IBS can be seen as a "stress disease" [36], and has been studied from different perspectives, at virtually all levels of the gut-brain-microbiome axis. There is evidence for HPA axis and autonomous nervous system involvement $[37,38]$, maladaptive coping and resilience [39-41], comorbidity of anxiety and depression [42], increased interoception [43] and altered neuronal pain processing $[44,45]$. Host-microbe interactions in the gut are important elements in the pathogenesis of IBS and other functional gastrointestinal diseases [46].

A variety of methods have been used to assess the human intestinal microbiota in IBS and this might be one reason (along with different diets, lifestyles, and geographical factors) why results are contradictory and difficult to summarize. Many studies have attempted to identify microbiota discriminating IBS patients from healthy controls, and to define dysbiosis in IBS and its sub-populations [47, 48]. However, a widely accepted concept of bacterial dysbiosis in IBS has not yet been established, since a large proportion of IBS patients display a "normal-like" microbiota profile $[49,50]$. Associations between clinical parameters and microbiota profiles assessed by classic ecological methods have remained largely elusive, but some bacteria stand out. A dysbalanced Firmicutes to Bacteroidetes ratio [49, 51], an increase in Clostridium XIVa and Ruminococcus [52], a reduction in Bifidobacterium [46, 52, 53], as well as a reduction in methanogens and butyrate- (a SCFA) producing bacteria in IBS with diarrhea (IBS-D) and IBS with diarrhea and constipation (mixed, IBS-M) patients [51, 54].

Some enterotypes and associations with clinical phenotypes have also been described: Prevotellapredominant enterotype seemed to be more common in healthy subjects, Bacteroides enterotype was more represented in IBS patients, while the Clostridiales-dominant enterotype was associated with faster colonic transit time. IBS severity could also be predicted by a complex microbial signature, consisting of bacterial families disseminated over the whole phylogenetic tree [54].

\section{Intestinal microbiota and psychological profiles in IBS}

Jeffery and colleagues [49], comparing the fecal microbiota of IBS patients with non-IBS individuals, separated their IBS cohort into three main clusters: a "normal-like" cluster with microbial characteristics highly similar to healthy controls and two "IBS clusters," the latter characterized by high Firmicutes to Bacteroidetes ratio. They also tested for associations between clinical variables and microbial composition, and found that depression was the single clinical feature that segregated in parallel with microbiota composition. Interestingly, depression was increased in patients belonging to the "normal-like" cluster.

Those findings are corroborated by Liu and colleagues [55], who observed that the fecal microbiome 
of IBS patients presented strong similarities to that of depressive patients. Sundin et al. [56] published data that distinguished the intestinal microbiota of postinfectious IBS patients from that of both general IBS patients and healthy controls. They also observed that altered fecal and mucosal microbial composition in post-infectious IBS patients correlated with psychological distress. Jeffery et al. [49] cautiously interpreted this pattern as more "physically-triggered" versus more "centrally-triggered" IBS. Our own data ${ }^{1}$ also strongly suggest that variables of psychological distress are associated with systematic microbial differences. Another recent study also found two clusters of IBS patients carrying either normal-like microbiomes or altered microbiomes. Here it was found that bacteria that carried metagenes involved in neurotransmitter metabolism correlated with morphological brain variations [57]. The pathways through which the gut microbiome affects the nervous system remain to be understood. However, some results point to western diet as a possible confounding factor: it affects the microbiota and its SCFA production, as well as the gut barrier integrity, and is associated with neuroinflammation and alteration of brain insulin sensitivity [58].

Therapeutic interventions are therefore possible from both ends of the spectrum: the brain and the gut microbiome. Antidepressants, psychotherapy and gut-directed hypnosis are effective, especially in refractory IBS [59, 60]. Until now, however, limited results are available from trials assessing the effectiveness of diet, prebiotics, probiotics, and antibiotics in IBS patients [61, 62].

\section{Conclusion}

The gut microbiota forms a crucial link in the bidirectional interactions between the intestine and the nervous system. Some alterations, like psychological distress or gastrointestinal infections, can affect these interactions and contribute to the development and/or affect the course of IBS. Symptomatic therapies such as IBS-type related medication, phytopharmacology, diet, probiotics and psychological interventions including gut directed hypnosis are recommended.

Further randomized clinical trials are needed to identify those IBS patients who will profit more from a) therapies that modulate the gut microbiome (prebiotics, probiotics, antibiotics), b) psychological interventions (psychotherapy, gut-directed hypnosis, antidepressants), or c) both approaches within an integrated psychosomatic care.

Acknowledgements Open access funding provided by Medical University of Vienna.

\footnotetext{
${ }^{1}$ Abstract accepted for presentation at the United Gastroenterology Week 2017: Peter et al., Psychological distress is associated with gut microbial differences in Irritable Bowel Syndrome.
}

Conflict of interest G. Moser, C. Fournier, and J. Peter declare that they have no competing interests.

Open Access. This article is distributed under the terms of the Creative Commons Attribution 4.0 International License (http://creativecommons.org/licenses/by/4.0/), which permits unrestricted use, distribution, and reproduction in any medium, provided you give appropriate credit to the original author(s) and the source, provide a link to the Creative Commons license, and indicate if changes were made.

\section{References}

1. Gill SR, Pop M, Deboy RT, et al. Metagenomic analysis of the human distal gut microbiome. Science. 2006;312(5778):1355-9.

2. ChoI, Blaser MJ. The human microbiome: at theinterface of health and disease. Nat Rev Genet. 2012;13(4):260-70.

3. Shreiner AB, KaoJY, YoungVB. Thegutmicrobiomein health and in disease. Curr Opin Gastroenterol. 2015;31(1):69-75.

4. Cryan JF, O'Mahony SM. The microbiome-gut-brain axis: from bowel to behavior. Neurogastroenterol Motil. 2011;23(3):187-92.

5. Kennedy PJ, Cryan JF, Dinan TG, et al. Kynurenine pathway metabolism and the microbiota-gut-brain axis. Neuropharmacology. 2017;112(PtB):399-412.

6. Clarke G, Grenham S, Scully P, et al. The microbiomegut-brain axis during early life regulates the hippocampal serotonergic system in a sex-dependent manner. Mol Psychiatry. 2013;18(6):666-73.

7. Sudo N, Chida Y, Aiba Y, et al. Postnatal microbial colonization programs the hypothalamic-pituitary-adrenal system for stress response in mice. J Physiol. 2004;558(Pt 1):263-75.

8. Feder A, Charney D, Collins K. Neurobiology of resilience. In: Southwick SM, Litz BT, Charney D, Friedman MJ, editors. Resilience and mental health: challenges across the lifespan. Cambrige: Cambrige University Press; 2011. pp.1-21.

9. McEwen BS, Gray J, Nasca C. Recognizing resilience: learning from the effects of stress on the brain. Neurobiol Stress. 2015;1:1-11.

10. Bharwani A, Mian MF, Foster JA, et al. Structural \& functional consequences of chronic psychosocial stress on the microbiome \& host. Psychoneuroendocrinology. 2016;63:217-27.

11. Galley JD, Bailey MT. Impact of stressor exposure on the interplay between commensal microbiota and host inflammation. Gut Microbes. 2014;5(3):390-6.

12. Dinan TG, Cryan JF. Regulation of the stress response by the gut microbiota: implications for psychoneuroendocrinology. Psychoneuroendocrinology. 2012;37(9):1369-78.

13. Moloney RD, O'Mahony SM, Dinan TG, et al. Stressinduced visceral pain: toward animal models of irritablebowel syndrome and associated comorbidities. Front Psychiatry. 2015;6:15.

14. O’Mahony SM, Marchesi JR, Scully P, et al. Early life stress alters behavior, immunity, and microbiota in rats: implications for irritable bowel syndrome and psychiatric illnesses. Biol Psychiatry. 2009;65(3):263-7.

15. Chang Y-M, El-Zaatari M, Kao JY. Does stress induce bowel dysfunction? Expert Rev Gastroenterol Hepatol. 2014;8(6):583-5.

16. Kelly JR, Kennedy PJ, Cryan JF, et al. Breaking down the barriers: the gut microbiome, intestinal permeability and stress-related psychiatric disorders. Front Cell Neurosci. 2015;9:392. 
17. Soderholm JD, Perdue MH. Stress and gastrointestinal tract. II. Stress and intestinal barrier function. Am J Physiol Gastrointest Liver Physiol. 2001;280(1):G7-G13.

18. Hall SB, Jones LK, Tyson L, et al. The inflammatory hypothesis of depression: implications for diagnosis and practice. J Ment Health Couns. 2016;38(2):124-38.

19. Maes M, Yirmyia R, Noraberg J, et al. The inflammatory \& neurodegenerative (I\&ND) hypothesis of depression: leads for future research and new drug developments in depression. Metab Brain Dis. 2009;24(1):27-53.

20. CaballeroS, PamerEG. Microbiota-mediatedinflammation and antimicrobial defense in the intestine. Annu Rev Immunol. 2015;33:227-56.

21. Maslowski KM, Mackay CR. Diet, gut microbiota and immune responses. Nat Immunol. 2011;12(1):5-9.

22. Richards JL, Yap YA, McLeod KH, et al. Dietary metabolites and the gut microbiota: an alternative approach to control inflammatory and autoimmune diseases. Clin Transl Immunology. 2016;5(5):e82.

23. Bercik P, Denou E, Collins J, et al. The intestinal microbiota affect central levels of brain-derived neurotropic factor and behavior in mice. Gastroenterology. 2011;141(2):599-609.e3.

24. Stilling RM, Dinan TG, Cryan JF. Microbial genes, brain \& behaviour - epigenetic regulation of the gut-brain axis. Genes Brain Behav. 2014;13(1):69-86.

25. Kiraly DD, Walker DM, Calipari ES, et al. Alterations of the host microbiome affect behavioral responses to cocaine. Sci Rep. 2016;6:35455.

26. Tillisch K, Labus J, Kilpatrick L, et al. Consumption of fermented milk product with probiotic modulates brain activity. Gastroenterology. 2013;144(7):1394-1401.e4.

27. Foster JA, McVey Neufeld K-A. Gut-brain axis: how the microbiome influences anxiety and depression. Trends Neurosci. 2013;36(5):305-12.

28. Goehler LE, Park SM, Opitz N, et al. Campylobacter jejuni infection increases anxiety-like behavior in the holeboard: possible anatomical substrates for viscerosensory modulation of exploratory behavior. Brain Behav Immun. 2008;22(3):354-66.

29. Zheng P, Zeng B, Zhou C, et al. Gut microbiome remodeling induces depressive-like behaviors through a pathway mediated by the host's metabolism. Mol Psychiatry. 2016;21(6):786-96.

30. Gacias M, Gaspari S, Santos P-MG, et al. Microbiotadriven transcriptional changes in prefrontal cortex override genetic differences in social behavior. eLife. 2016; doi:10. 7554/elife.13442.

31. Lin P, Ding B, Feng C, et al. Prevotella and Klebsiella proportions in fecal microbial communities are potential characteristic parameters for patients with major depressive disorder. JAffect Disord. 2017;207:300-4.

32. Jiang $\mathrm{H}$, Ling $\mathrm{Z}$, Zhang $\mathrm{Y}$, et al. Altered fecal microbiota composition in patients with major depressive disorder. Brain Behav Immun. 2015;48:186-94.

33. Kleiman SC, Bulik-Sullivan EC, Glenny EM, et al. The gutbrain axis in healthy females: lack of significant association between microbial composition and diversity with psychiatric measures. PLOS ONE. 2017;12(1):e0170208.

34. Drossman DA. The functional gastrointestinal disorders and the Rome III process. Gastroenterology. 2006;130(5):1377-90.

35. Mearin F, Lacy BE, Chang L, et al. Bowel disorders. Gastroenterology. 2016;150(6):1393-1407.e5.

36. Mayer EA, Naliboff BD, Chang L, et al. V. Stress and irritable bowel syndrome. Am J Physiol Gastrointest Liver Physiol. 2001;280(4):G519-G24.
37. Kennedy PJ, Cryan JF, Quigley EMM, et al. A sustained hypothalamic-pituitary-adrenal axis response to acutepsychosocial stress in irritable bowel syndrome. Psychol Med. 2014;44(14):3123-34.

38. Pellissier S, Dantzer C, Mondillon L, et al. Relationship between vagal tone, cortisol, TNF-alpha, epinephrine and negative affects in Crohn's disease and irritable bowel syndrome. PLOSONE. 2014;9(9):e105328.

39. Kilpatrick LA, Gupta A, Love AD, et al. Neurobiology of psychological resilience in irritable bowel syndrome (IBS) and inflammatory bowel disease (IBD) patients. Gastroenterology. 2015;148(4):S-774. Abstr Mo2030.

40. Knowles SR, Austin DW, Sivanesan S, et al. Relations between symptom severity, illness perceptions, visceral sensitivity, coping strategies and well-being in irritable bowel syndrome guided by the common sense model of illness. Psychol Health Med. 2017;22(5):524-34.

41. Stanculete MF, Matu S, Pojoga C, et al. Coping strategies and irrational beliefs as mediators of the health-related quality of life impairments in irritable bowel syndrome. JGastrointest Liver Dis. 2015;24(2):159-64.

42. Fond G, Loundou A, Hamdani N, et al. Anxiety and depression comorbidities in irritable bowel syndrome (IBS): a systematic review and meta-analysis. Eur Arch Psychiatry Clin Neurosci. 2014;264(8):651-60.

43. Moloney RD, Johnson AC, O'Mahony SM, et al. Stress and the microbiota-gut-brain axis in visceral pain: relevance to irritable bowel syndrome. CNS Neurosci Ther. 2016;22(2):102-17.

44. Claassen J, Labrenz F, Ernst TM, et al. Altered cerebellar activity in visceral pain-related fear conditioning in irritable bowel syndrome. Cerebellum. 2016;16(2):508-17.

45. Schmid J, Langhorst J, Gass F, et al. Placebo analgesia in patients with functional and organic abdominal pain: a fMRI study in IBS, UC and healthy volunteers. Gut. 2015;64(3):418-27.

46. Simren M, Barbara G, Flint HJ, et al. Intestinal microbiota in functional bowel disorders: a Rome foundation report. Gut. 2013;62(1):159-76.

47. Casen C, Vebo HC, Sekelja M, et al. Deviations in human gut microbiota: a novel diagnostic test for determining dysbiosis in patients with IBS or IBD. Aliment Pharmacol Ther. 2015;42(1):71-83.

48. Jalanka J, Salonen A, Fuentes S, et al. Microbial signatures in post-infectious irritable bowel syndrome--toward patient stratification for improved diagnostics and treatment. Gut Microbes. 2015;6(6):364-9.

49. Jeffery IB, O'Toole PW, Ohman L, et al. An irritable bowel syndrome subtype defined by species-specific alterations in faecal microbiota. Gut. 2012;61(7):997-1006.

50. Jeffery I, O'Toole P, Fraher M, et al. Alterations in the microbiota in irritable bowel syndrome; a comparison of two geographically distinct cohorts. Gastroenterology. 2016;150(4):S63. Abstr 260.

51. Pozuelo M, PandaS, Santiago A, etal. Reduction ofbutyrateand methane-producing microorganisms in patients with Irritable Bowel Syndrome. Sci Rep. 2015;5:12693.

52. Rajilic-Stojanovic M, Jonkers DM, Salonen A, et al. Intestinal microbiota and diet in IBS: causes, consequences, or epiphenomena? Am J Gastroenterol. 2015;110(2):278-87.

53. Mayer EA, Savidge T, Shulman RJ. Brain-gut microbiome interactions and functional bowel disorders. Gastroenterology. 2014;146(6):1500-12.

54. Tap J, Derrien M, Tornblom H, et al. Identification of an intestinal microbiota signature associated with severity of irritable bowel syndrome. Gastroenterology. 2017;152(1):111-123.e8. 
55. Liu Y, Zhang L, WangX, et al. Similar fecal microbiota signatures in patients with diarrhea-predominantirritable bowel syndrome and patients with depression. Clin Gastroenterol Hepatol. 2016;14(11):1602-1611.e5.

56. Sundin J, Rangel I, Fuentes S, et al. Altered faecal and mucosal microbial composition in post-infectious irritable bowel syndrome patients correlates with mucosal lymphocyte phenotypes and psychological distress. Aliment Pharmacol Ther. 2015;41(4):342-51.

57. Labus JS, Hollister EB, Jacobs J, et al. Differences in gut microbial composition correlate with regional brain volumes in irritable bowel syndrome. Microbiome. 2017;5(1):49.

58. Noble EE, Hsu TM, Kanoski SE. Gut to brain dysbiosis: mechanisms linking western diet consumption, the microbiome, and cognitive impairment. Front Behav Neurosci. 2017;11:9.
59. Enck P, Junne F, Klosterhalfen S, et al. Therapy options in irritable bowel syndrome. Eur J Gastroenterol Hepatol. 2010;22(12):1402-11.

60. Moser G, Tragner S, Gajowniczek EE, et al. Long-term success of GUT-directed group hypnosis for patients with refractory irritable bowel syndrome: a randomized controlled trial. Am J Gastroenterol. 2013;108(4):602-9.

61. Dapoigny M, Piche T, Ducrotte P, et al. Efficacy and safety profile of LCR35 complete freeze-dried culture in irritable bowel syndrome: a randomized, double-blind study. World J Gastroenterol. 2012;18(17):2067-75.

62. Han K, Wang J, Seo J-G, et al. Efficacy of double-coated probiotics for irritable bowel syndrome: a randomized doubleblind controlled trial. J Gastroenterol. 2016;52(4):432-43. 